\title{
Video Technique on Medial Open Reduction for DDH
}

\author{
Premal Naik ${ }^{1,2}$ \\ Received: 9 August 2021 / Accepted: 22 August 2021 \\ (c) Indian Orthopaedics Association 2021
}

\begin{abstract}
Background The most common approach for the open reduction of DDH is the anterolateral approach. After an initial report by Weinstein and Ponseti, the medial approach for DDH has garnered great interest.

Purpose The medial approach for DDH allows easy access to the structures which block the reduction of the femoral head into the acetabulum; namely the psoas tendon, inferior capsule, and ligamentum teres. It uses a skin crease small incision in the groin with excellent cosmetic outcome and is very well hidden.

Method The classical medial approach described by Ludloff uses interval between the adductor brevis and pectineus. The anteromedial approach to the hip uses the same incision but interval between the pectineus and femoral neurovascular bundle and allows better handling of medial circumflex vessels. A technical modification suggested by Late Ian Torode involves tenodesis of the ligamentum teres which improves the stability of the hip reduction. This video demonstrates the medial approach for the open reduction of DDH in a fourteen months old girl with medium-term follow-up results. The technique of the tenodesis of ligamentum teres is also shown.

Result The medial approach is safe with a low rate of re-dislocation; it does not violate the hip abductors and iliac apophysis. The major disadvantage of the medial approach is the restricted operative field and a higher rate of AVN. Many authors have found the rate of AVN comparable to other approaches and the grade of AVN milder culminating in good long-term results. Conclusion We have found this approach very useful in children with DDH, before they start walking (when the closed reduction is unstable) and arthrogrypotic children with hip dislocation.
\end{abstract}

Keywords Ludloff approach $\cdot$ Medial open reduction $\cdot$ DDH $\cdot$ Ligamentum teres tenodesis $\cdot$ Arthrogrypotic DDH

Supplementary Information The online version contains supplementary material available at https://doi.org/10.1007/s43465-021-00502-6.

\section{Declarations}

Conflict of interest Author does not have any conflict of interest.

Ethical Approval This article does not contain any studies with human or animal subjects performed by the any of the authors.
Informed Consent For this type of study informed consent is not required.
Publisher's Note Springer Nature remains neutral with regard to jurisdictional claims in published maps and institutional affiliations.

Premal Naik

premal_naik@yahoo.com

1 Rainbow Superspeciality Hospital and Children's Orthopaedic Centre, Next To Asia School, Behind HDFC Bank, Opposite Drive in Cinema, Bodakdev, Ahmedabad, Gujarat 380 054, India

2 Smt S C L Hospital, NHL Municipal Medical College, Ahmedabad, Gujarat, India 